\title{
Neurotrophic keratitis secondary to dorsal medullary infarction
}

\author{
Gairik Kundu', Thanuja GP²* \\ ${ }^{1}$ Junior Resident, ${ }^{2}$ Assistant Professor, Dept. of Ophthalmology, Ramaiah Medical College, Bangalore, Karnataka, India
}

\section{Article Info}

Received: $12^{\text {th }}$ November, 2018

Accepted: $11^{\text {th }}$ April, 2019

Published Online: $9^{\text {th }}$ September, 2019

Keywords: Neurotrophic keratitis, Persistent epithelial defect, Stroke.

\begin{abstract}
Introduction: To report three cases with neurotrophic keratitis secondary to cerebrovascular accident and to highlight the importance of eliciting the history of previous stroke and their details in order to identify the cause of neurotrophic keratitis.

Materials and Methods: We present three cases of neurotrophic keratitis which presented many years later after a cerebro-vascular accident. The cases were misdiagnosed and treated as infectious keratitis earlier and the healing of epithelial defect posed a challenge. All the cases required surgical intervention to stabilize the eye and save the globe.

Results: The three cases of neurotrophic keratitis which were not responding to standard care of treatment and presented with persistent epithelial defect, which on enquiry revealed all patients had a previous history of stroke.

Conclusion: Cases of cerebrovascular accidents especially those who have medullary infarction require life long follow-up as corneal affection may develop many years later. These cases require a correct elucidation of history to arrive at the right diagnosis in order to initiate the appropriate treatment and ensure reduced morbidity.
\end{abstract}

\section{Introduction}

The corneal sensory nerves are crucial for the integrity of the corneal epithelium, and their loss affects the metabolism and mitotic activity of the epithelial cells thus reducing their viability. The histopathological changes reveal epithelial intracellular swelling, loss of microvilli and also affects the production of basal lamina. These changes make the eye susceptible to even minor trauma and hence vulnerable to infection. Despite the presence of intact blink reflex and normal tear production, the epithelial breakdown continues.

The causes of neurotrophic keratitis can be local causes such as previous corneal surgery, trauma, ocular anaesthetic abuse, long term use of topical drugs, chemical injury, previous herpes simplex or herpes zoster infection and certain corneal dystrophies. Identification of these ocular associations are easy for an Ophthalmologist, however neurotrophic keratitis can be due to central causes which include cerebrovascular accidents, brain tumors and thromboangitis obliterans. These are equally important casues and require due consideration while examining a neurotrophic keratitis.

We report three cases of neurotrophic kerattiis secondary to a cerebrovascular event and presented with a varied presentation to the ophthalomology department.
Patient had no history of diabetes or hypertension. He was a smoker who had quit smoking. Patient was not improving with treatment and continued to have PED. On examination patient was seen to have OD, visual acuity $6 / 6$, normal anterior segment and posterior segment. OS, visual acuity was counting fingers $(\mathrm{CF})$ at $2 \mathrm{~m}$, a medial tarsorraphy done with suture edges rubbing on the cornea. Corneal examination revealed a central $5 * 7 \mathrm{~mm}$ epithelial defect (Fig. 1), absent corneal sensations, rest of the anterior segment had no abnormality and fundus was normal. The patient was relatively comfortable despite a huge PED. On examination he had absent corneal sensation and also sensations over ipsilateral forehead were reduced. On further questioning patient revealed history of stroke 15 years back and said he had recovered completely. The sutures rubbing on the cornea were removed and patient was treated with lubricants and gel. He had improvement with reduction in epithelial defect but failed to have complete resolution. Patient was advised amniotic membrane transplantation (AMT) but refused and hence managed with daily patching. Patient showed an initial response but the epithelial defect would break down causing repeated epithelial defect. Patient was then lost for follow-up.

\section{Case 1}

A 70 -year old male patient presented with persistent epithelial defect (PED) of cornea left eye since 1 month for which he was treated elsewhere with temporary tarsorraphy.

\footnotetext{
*Corresponding Author: Thanuja GP, Assistant Professor, Dept. of Ophthalmology, Ramaiah Medical College, Bangalore, Karnataka, India Email: thanugopal@yahoo.co.in http://doi.org/10.18231/j.ijceo.2019.100
} 


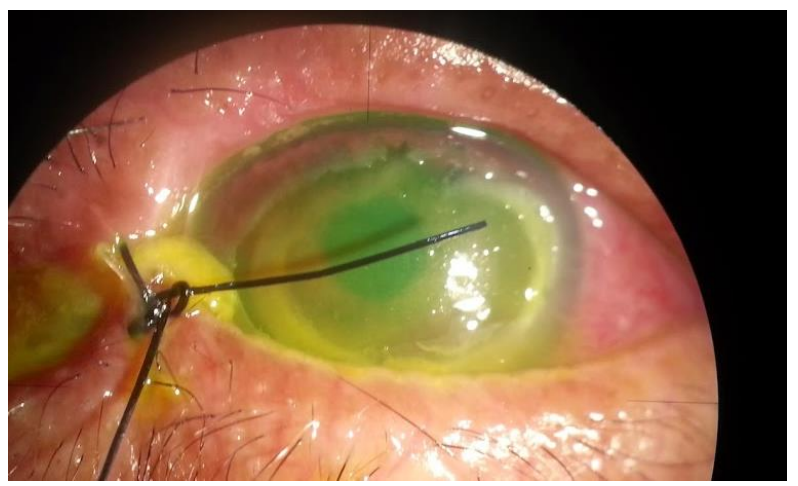

Fig. 1: Picture showing a central corneal $5 * 7 \mathrm{~mm}$ epithelial defect post a medial tarsorraphy

\section{Case Report 2}

A 65 year old male patient came with history of redness and watering in left eye since two months. Patient had undergone treatment initially for bacterial and then for fungal keratitis with no improvement. When we examined him his OD was normal with vision 6/6, anterior segment and fundus normal. OS showed perception of light. He had 360 degree vascularization, a central epithelial defect of $5 *$ $5 \mathrm{~mm}$ (Fig. 2), stromal infiltrates in superior margin with inferior margin showing scarring, $\mathrm{He}$ had posterior synechiae and cataractous lens. Corneal sensations were absent and he also had diminished sensations over left side of forehead. He revealed a history of stroke 12 years back and had completely recovered from it. Old records showed he was diagnosed with lateral medullary syndrome. Patient was treated with BCL and preservative free lubricants. The epithelial defect reduced in size slowly and hence he was taken up for AMT. The ocular surface stabilized with it and he had a faint central scar at the end of 12 months.

\section{Case Report 3}

A 70 year old male patient presented with redness and watering left eye since 6 months. He had no pain. Right eye he had similar complaints and patient was treated elsewhere with bandage contact lens (BCL) 6 months back. On examination he had OD vision of perception of light with mature cataract and corneal leucomatous scarring involving temporal cornea with superficial vascularization. Left eye he had an epithelial defect involving the infero-temporal cornea (Fig. 3) with superficial vascularization and immature cataract left eye. CNS examination was normal. He was treated with AMT and showed improvement. Patient gave history of stroke 6 years back and had completely recovered from it.
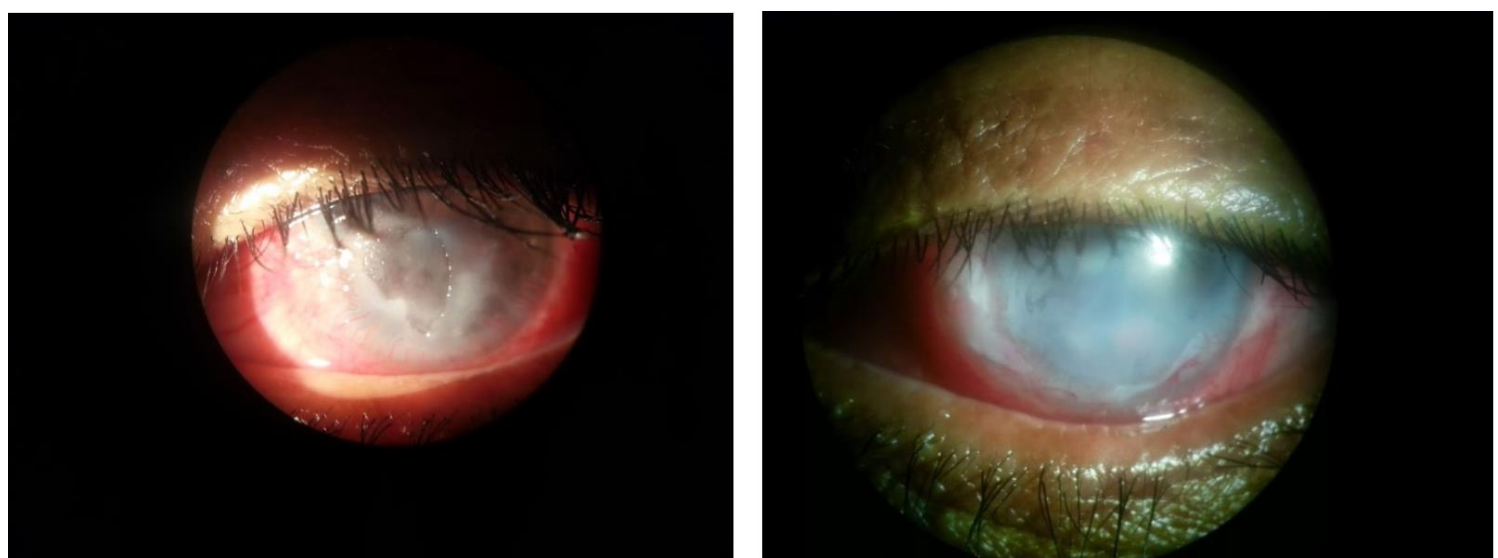

Fig. 2: Picture showing 360 degree corneal vascularization with a central epithelial defect of $5 * 5 \mathrm{~mm}$ and stromal infiltrates in superior margin with inferior margin showing scarring. Patient after amniotic membrane graft.
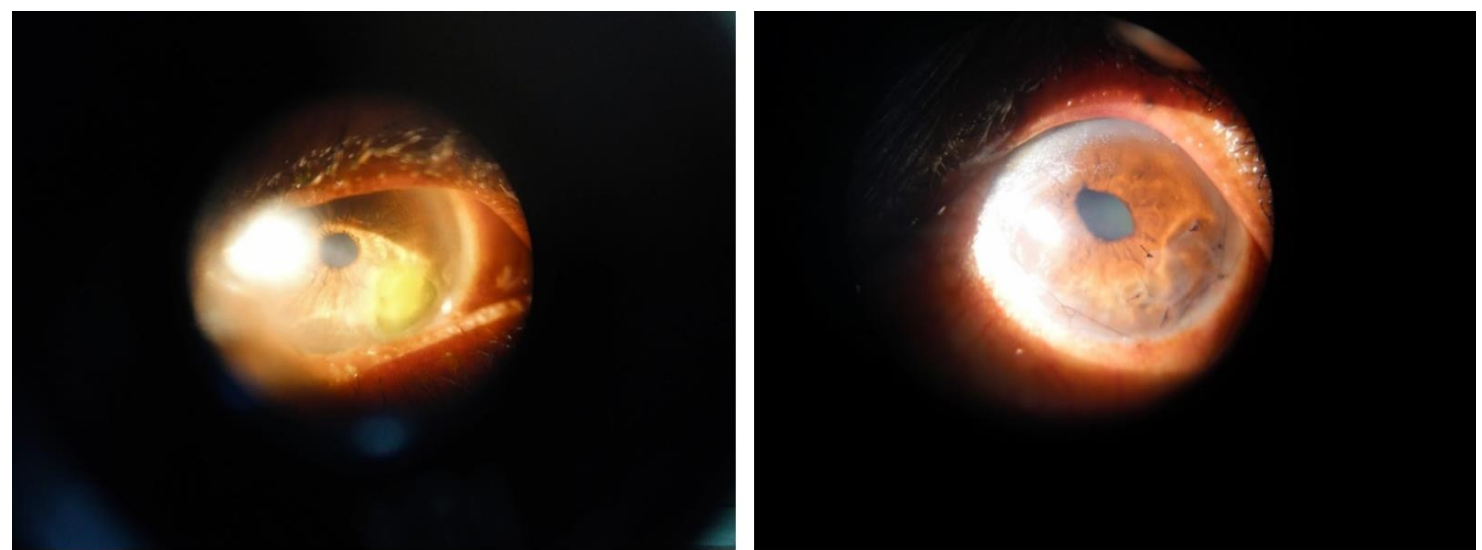

Fig. 3: Picture showing epithelial defect involving the infero-temporal cornea with superficial vascularization. Post AMT picture 


\section{Discussion}

Corneal injury secondary to stroke such as in Wallenberg syndrome is a rare cause of unilateral keratitis, as is reported by $\mathrm{Wu}$ et $\mathrm{al}^{1}$ and Hipps et $\mathrm{al}^{2}$ In these cases they report that neurotrophic keratitis secondary to dorsal medullary infarction affecting the spinal trigeminal tract or nuclei are characterised by distinct features of persistent ipsilateral facial hypolgesia and thermohypesthesia. There is delayed onset time of corneal damage (e.g. one to two months after dorsolateral medullary infarction) and the corneal disease shows a rapid progression with relatively serious clinical manifestations such as corneal ulcers. The neurotrophic keratitis management in these cases is difficult and has poor prognosis despite aggressive treatment.

The signs of neurotrophic keratitis may present years after an ischemic event as shown in our three cases and have various manifestations, such as corneal erosions, persistent epithelial defects/ulcerations, and even hypopyon, which can mimic infectious keratitis. ${ }^{3}$

Neurotrophic keratitis secondary to central causes (i.e., stroke) may present initially with erosions which may be asymptomatic to the patient. Early detection of this condition at this stage is very important to prevent progression and development of secondary corneal infection and abscess formation. The neurotrophic keratitis due to central causes are resilient to treatment and the treatment is usually time-consuming and costly hence early detection and treatment remains crucial in their management. As a result, patients with Wallenberg syndrome should undergo comprehensive ophthalmologic examinations, including slitlamp examination, corneal esthesiometer, and Schirmer's test, to rule out trigeminal nerve injury and to grade severity of anterior segment damage. ${ }^{4}$ In these patients, the ophthalmologist should consider prophylactic treatment, which include lubricating eye drops and a regular follow up. Therapeutic measures in cases of punctate keratitis include the daily periodic application of artificial tears, lubricating gels and autologous serum. ${ }^{5}$ Additional procedures such as punctal plugs, amniotic membrane transplantation, ${ }^{6,7}$ and surgical tarsorrhaphy may become necessary if the disease worsens and should be considered early in the disease management.

Multidisciplinary management is required for these patients. Neurology specialists should contemplate this pathology in order to refer these patients to the ophthalmology department.

\section{Conclusion}

The diagnosis and management of NK is a challenge for ophthalmologists. The currently available medical and surgical treatments aim to promote healing, prevent disease progression, and avoid corneal perforation. However, no therapies are currently available to improve the impairment of corneal sensitivity and restore visual acuity. Patients with stroke such as in Wallenberg syndrome should undergo a regular ophthalmological evaluation to facilitate early diagnosis and ensure proper medical management. Ophthalmologists also should take careful patient histories and should consider neurological causes of neurotrophic keratitis.

Source of Funding: None.

Conflict of Interest: None.

\section{References}

1. Hipps WM, Wilhelmus KR: Persistent visual loss from neurotrophic corneal ulceration after dorsolateral medullary infarction (Wallenberg syndrome). J Neuroophthalmol 2004;24:345-6.

2. Neurotrophic keratopathy due to dorsolateral medullary infarction (Wallenberg Syndrome): case report and literature review Wu et al. BMC Neurol 2014;14:231.

3. Bonini S, Rama P, Olzi D, Lambiase A. Neurotrophic keratitis. Eye 2003;17:989-95.

4. Affeldt JC. Neurotrophic keratitis and persistent epithelial defect. In: Yiu SC, ed. Epithelial cell biology: implications for the ocular surface. Los Angeles: Doheny Eye Institute, 2003:13-6.

5. Jeng BH, Dupps WJ Jr. Autologous serum $50 \%$ eye drops in the treatment of persistent corneal epithelial defects. Cornea 2009;28:1104-8.

6. Chen HJ, Pires RT, Tseng SC. Amniotic membrane transplantation for severe neurotrophic corneal ulcers. $\mathrm{Br} J$ Ophthalmol 2000;84:826-33.

7. Prabhasawat P, Tesavibul N, Komolsuradej W. Single and multilayer amniotic membrane transplantation for persistent corneal epithelial defect with and without stromal thinning and perforation. Br J Ophthalmol 2001;85:1455-63.

How to cite this article: Kundu G, Thanuja GP. Neurotrophic keratitis secondary to dorsal medullary infarction. Indian J Clin Exp Ophthalmol 2019;5(3):4146. 